\title{
General Scattering Mechanism and Transport in Graphene
}

\author{
Musah Rabiu ${ }^{1}$, Samuel Y. Mensah ${ }^{2}$, Sulemana S. Abukari ${ }^{2}$ \\ ${ }^{1}$ Department of Applied Physics, Faculty of Applied Science, University for Development Studies, Navrongo, Ghana \\ ${ }^{2}$ Center for Laser and Fiber Optics, Physics Department, University of Cape Coast, Cape Coast, Ghana \\ Email:mrabiu@uds.edu.gh
}

Received November 14, 2012; revised December 19, 2012; accepted January 17, 2013

\begin{abstract}
Using quasi-time dependent semi-classical transport theory, within relaxation time approximation, we obtained coupled electronic current equations in the presence of time varying field, and based on general scattering mechanism, $\tau \propto \varepsilon^{\beta}$. In the vicinity of Dirac points, we find that a characteristic exponent $\beta=+2$ corresponds to acoustic phonon scattering, $\beta=+1$ long-range Coulomb scattering mechanism and $\beta=-1$ is short-range (delta or contact potential) scattering in which the conductivity is constant of temperature. The $\beta=0$ case is the ballistic regime. In the low-energy dynamics of Dirac electrons in graphene, the effect of the time-dependent electric field is to alter just the electron charge by $e \rightarrow e /\left(1+i \Omega \tau_{0}\right)$ making electronic conductivity non-linear. The effect of constant magnetic field at finite temperature is also considered.
\end{abstract}

Keywords: Boltzmann Transport Equation; Relaxation Time Approximation; Graphene Energy Spectrum; Electronic Conductivity; Scattering

\section{Introduction}

Quite recently, semiconductor nanostructures have become the model of choice for investigation of electrical conduction. The unique two-dimensional material graphene which was first thought to be an academic material is not an exception. This 2D nanomaterial is fast becoming better candidate for electronic devices. Not only because of its noble electronic transport properties [1], but it also promises a good future in graphene based electronics industry. Graphene has received a wide academic attention and serve as a bridge between condensed matter physics and high energy physics [2]. The advantage of this planner material is that, one can easily change its electronic properties by introducing tunable gap in the sample or changing the number of graphene planes [3]. It is also possible to fabricate free standing graphene sheets [4]. Intrinsic superconducting states can also be realized in graphene [5]. These among other things, means that the electronic properties of graphene can easily be tailored to fit device conditions.

The crystal structure of graphene is made up of monolayer of carbon atoms arranged in hexagonal lattice. The low energy dynamics of fermions in graphene is characterized by linear dispersion, $\mathcal{E}(k) \sim v_{F}|p|$. In a cleaned sample, the conduction and valence bands touch at two inequivalent Dirac points located at the corners of Brillouin zone. To understand the low energy transport in graphene, a relevant scattering potential (mechanism) is essential. However, as we shall see in this report, one does not need to consider any explicit form of scattering potential. It was shown in [6] that Boltzmann theory with long-range coulomb scattering can account for all experimental findings. Especially, when the electronic density around Dirac points are normalized. Also, within the Boltzmann theory using random phase approximation, coulomb scattering has been predicted to be the dominant scattering mechanism [7]. Several theories including Boltzmann Transport Equation (BTE) suggest a nonuniversal behavior of minimal conductivity which nonetheless coincides with experimentally observed value times, i.e. $\pi, \sigma_{\text {thory }}=\sigma_{\text {exp }} / \pi[8,9]$. The same BTE predict other transport coefficients which agree well with experiment [9-11].

In this brief report, we reproduce transport properties of graphene. Within the BTE formalism and with energy dependent relaxation time depending on power law, we showed dependence of graphene's transport coefficients on applied field frequency. The remaining of this paper is organized as follows: Section 2 formulates BTE and provides arguments leading to a quasi-time dependent ( $t$ BTE) solution. In Section 3 we used the t-BTE to derive coupled current equations from which we derived conductivity and other transport quantities. The conductivity tensor is re-derived in the presence of magnetic. The last 
Section 4 contains discussion, conclusion and some recommendations.

\section{BTE and Quasi-Time Dependent Solution}

A time time-dependent linearized BTE has the following form [12]

$$
\begin{aligned}
& \partial_{t} f(p)+v(p) \cdot\left[-\frac{\varepsilon-\mu}{T} \nabla_{r} T+e \nabla_{r}\left(\frac{\mu}{e}\right)\right]\left(-\frac{\partial f(p)}{\partial \varepsilon}\right), \\
& =\mathcal{C} f(p)
\end{aligned}
$$

where $f(p)$ depends on $t, r$ and $p$, i.e. $f(t, r, p)$ The group velocity, $v$ is constant of time, $\mathcal{C} f(p)$ is the scattering term and $T$ is the lattice temperature. The applied electric field has the form $E(t)=E_{0} \cos (\Omega t)$, $E_{0}$ is the static electric field. Exact analytical solution of (1) is very difficult to obtain. Especially, the non-linearity of the scattering term and the fact that velocity can generally depend on time. In view of this, we adopt some approximations including relaxation time approximation where

$$
\mathcal{C} f(p)=-\Gamma(\varepsilon)\left(f(p)-f_{0}(p)\right) .
$$

$\Gamma$ is inverse of relaxation time $\tau . f$ and are $f_{0}$ the time-dependent (equilibrium) and time-independent (nonequilibrium) Fermi-Dirac distribution functions. Motivated by [13] in the absence of magnetic field $(B=0)$, we consider a picture where the only time dependent quantity in (1) is the electric field. Note that Mensah solution considered the space term as perturbation. Under the above simplified assumptions together with the steady state solution $[12,14]$, the quasi solution is

$$
\begin{aligned}
f(p)= & \Gamma \int_{0}^{\infty} \mathrm{d} t \mathrm{e}^{\Gamma t^{\prime}} f_{0}(p)-e \int_{0}^{\infty} \mathrm{d} t \mathrm{e}^{\Gamma t^{\prime}} v(p) \\
& \cdot\left[-\frac{\varepsilon-\mu}{e T} \nabla_{r} T+\nabla_{r}\left(\frac{\mu}{e}\right)-E\left(t^{\prime}\right)\right] \times\left(-\frac{\partial f(p)}{\partial \varepsilon}\right)
\end{aligned}
$$

so that it can easily reduce back to [13] when $\Omega=0$.

\subsection{Coupled Currents and Transport Coefficients}

The sheet current for electron and energy flux in graphene are defined by the formulas

$$
J_{e}=\frac{g_{s} g_{v} e}{A} \sum_{v} v(p) f(p)
$$

and

$$
J_{\varepsilon}=\frac{g_{s} g_{v} e}{A} \sum_{p} \varepsilon(p) f(p) .
$$

Where $g_{s}, g_{v}$ are spin and valley degeneracies and $A$ is graphene sheet area. We convert the sums in (4) and (5) to integrals following

$$
\sum_{p} \rightarrow \frac{A}{(2 \pi \hbar)^{2}} \int_{-\pi}^{\pi} \mathrm{d} \theta \int_{0}^{\infty} p \mathrm{~d} p .
$$

Substituting (2) in to (3), (4) and simplifying using an energy dependent relaxation time of the form

$$
\tau(\varepsilon)=\Lambda \varepsilon^{\beta},
$$

where $A$ is constant of energy with dimensions of $s / J^{\beta}$ and $\beta$ is characteristic exponent which determines the specific type of scattering mechanism involved. One easily obtains coupled current equations

$$
\begin{gathered}
J_{e}=\sigma(\Omega)\left(-\nabla_{r} \Phi\right)+S\left(-\nabla_{r} T\right) \\
J_{\varepsilon}=T S\left(-\nabla_{r} \Phi\right)+K\left(-\nabla_{r} T\right) .
\end{gathered}
$$

Where the measured electrochemical potential gradient, $\nabla \Phi=\nabla_{r}(\mu / e)-E$. The coefficients in (5) are

$$
\begin{gathered}
\sigma_{\beta}(\Omega)=\frac{\sigma_{\min }}{u_{0}^{2}} \frac{\mu^{\beta+1}+\frac{\pi^{2}}{6}\left(k_{B} T\right)^{2} \beta(\beta+1) \mu^{\beta-1}+\cdots}{1-i \Omega \Lambda \mu} \\
S_{\beta}(\Omega)=\frac{\sigma_{\min }}{u_{0}^{2}} \frac{\pi^{2}}{3 e T}\left(k_{B} T\right)^{2}(\beta+1) \mu^{\beta}+\cdots \\
K_{\beta}(\Omega)=\frac{\sigma_{\min }}{u_{0}^{2}} \frac{\pi^{2}}{3 e T}\left(k_{B} T\right)^{2} \mu^{\beta+1}+\cdots
\end{gathered}
$$

$\sigma_{\min }=2 e^{2} / h$ is the minimal conductivity in graphene and the constant $u_{0}$ is defined through $u_{0}^{2}=\hbar / \Lambda$. It has dimension of energy square.

Now, to derive a particular type of scattering mechanism, we consider specific cases when $\tau \propto \varepsilon^{2}, \tau \propto \varepsilon$, $\tau \propto 1 / \varepsilon$ and $\tau \sim$ constant which correspond to $\beta=$ $+2,+1,-1,0$ respectively. Because the electronic conductivity, $\sigma$ is the only coefficient depending on frequency, we specifically study this quantity for various $\beta$ values. For $\beta=-1$, the conductivity in (8) assumes the form

$$
\sigma_{-1}(\Omega)=\frac{\sigma_{\min }}{u_{0}^{2}} \frac{1}{1+(\Omega \Lambda \mu)^{2}} .
$$

This is characterized by short-range potential that has the form of contact (or delta) potential and may be due to localized impurity (defect) in the sample [9,10,15]. For $\beta=0$, we get

$$
\sigma_{0}(\Omega)=\frac{\sigma_{\min }}{u_{0}^{2}} \frac{\mu}{1+(\Omega \Lambda \mu)^{2}},
$$

which corresponds to coherent [9] or random Dirac mass scattering, and describes the ballistic scattering for electronic conductivity in graphene. Behavior of the electronic conductivity, $\sigma_{0}$ resulting from these processes 


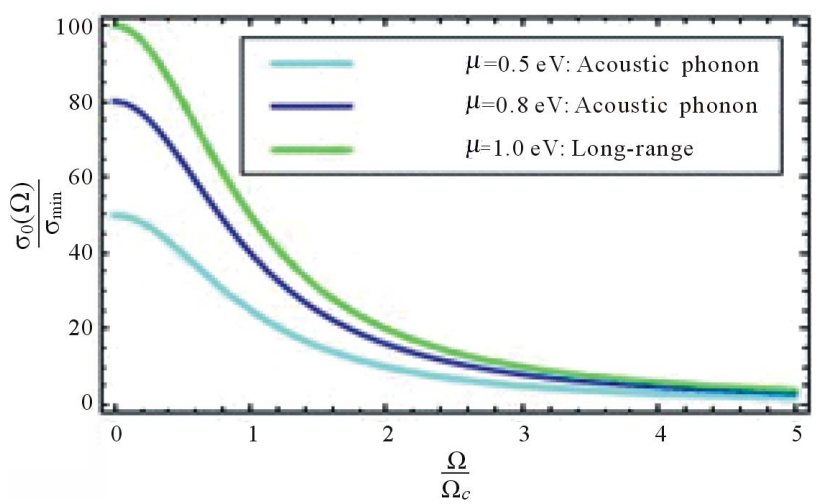

Figure 1. $\beta=0$ : Normalized conductivity is plotted against $\Omega / \Omega_{c}$ at fixed values of doping; $\mu=1.0 \mathrm{eV}, 0.8 \mathrm{eV}, 0.5 \mathrm{eV}$ with $K_{B} T=0.1 \mathrm{eV}$ and $\alpha=2.3 \mathrm{~m}^{2} \cdot \mathrm{V}^{-1} \cdot \mathrm{s}^{-1}$.

is shown in Figure 1 at fixed values of chemical potential (doping). Finally, for $\beta=+1$, the electronic conductivity becomes

$$
\sigma_{+1}(\Omega)=\frac{\sigma_{\min }}{u_{0}^{2}} \frac{\mu^{2}+\frac{\pi^{2}}{3}\left(k_{B} T\right)^{2}}{1+(\Omega \Lambda \mu)^{2}} .
$$

This is an important and dominant scattering mechanism in graphene [16]. It is characterized by unscreened long-range Coulomb (charged impurity) scattering [7]. The second term in (13) is inevitable at finite temperatures. This extra term was missing in [9]. It is the contribution due to scattering by phonons. From (13) conductivity departs slightly from linearity behavior at low frequencies. Figure 2 depicts this situation.

The conductivity for acoustic phonon scattering is identified with $\beta=+2$,

$$
\sigma_{+2}(\Omega)=\frac{\sigma_{\min }}{u_{0}^{2}} \frac{\mu^{3}+\pi^{2}\left(k_{B} T\right)^{2} \mu}{1+(\Omega \Lambda \mu)^{2}} .
$$

\subsection{Resistivity, Thermal Conductivity and Thermopower}

In this section we turn to (7) to compute other transport properties of graphene. Specifically, we will calculate the resistivity $\rho$, thermal conductivity, $\kappa$ and thermopower, $S_{0}$ for $\beta=+1$ We will drop the $\beta$ subscript in the following equations. By inverting (7), one can find these quantities that experimentalist usually like working with. We will write our equations similar to the format in [9]. The resistivity is,

$$
\rho(\Omega)=\frac{u_{0}^{2}}{\sigma_{\min }} \frac{1+(\Omega \Lambda \mu)^{2}}{\mu^{2}+\frac{\pi^{2}}{3}\left(k_{B} T\right)^{2}},
$$

thermal conductivity

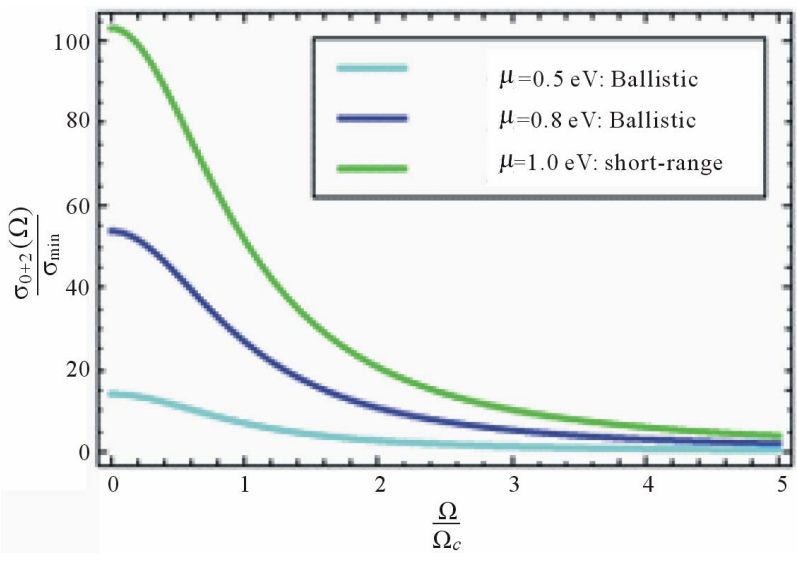

Figure 2. $\beta=+2$ : Normalized conductivity is plotted against $\Omega / \Omega_{c}$ at fixed values of doping; $\mu=1.0 \mathrm{eV}, 0.8 \mathrm{eV}, 0.5 \mathrm{eV}$ with $K_{B} T=0.1 \mathrm{eV}$ and $\alpha=2.3 \mathrm{~m}^{2} \cdot \mathrm{V}^{-1} \cdot \mathrm{s}^{-1}$.

$$
\begin{aligned}
\kappa(\Omega)= & \frac{2}{3} \frac{\pi^{2}}{h T}\left(k_{B} T\right)^{2} \mu^{2} \\
& -\frac{8}{9} \frac{\pi^{4}}{h T}\left(K_{B} T\right)^{4} \frac{1+(\Omega \Lambda \mu)^{2}}{1+\frac{\pi^{2}}{3} \frac{\left(k_{B} T\right)^{2}}{\mu^{2}}}
\end{aligned}
$$

and thermoelectric power

$$
S_{0}(\Omega)=\frac{2}{3} \frac{\pi^{2}}{e T} \frac{\left(K_{B} T\right)^{2}}{\mu} \frac{1+(\Omega \Lambda \mu)^{2}}{1+\frac{\pi^{2}}{3} \frac{\left(k_{B} T\right)^{2}}{\mu^{2}}} .
$$

The new physics emerging from these equations is the linear dependence of these quantities on $\Omega^{2}$. Note that electron density $n$ dependence in our equations is self manifest, since one can easily incorporate it through [9] $n \propto \mu^{2}$ at zero temperature or

$$
n(T)=\frac{1}{\pi} \frac{1}{\left(\hbar v_{F}\right)^{2}}\left(\mu^{2}+\frac{\pi^{2}}{3}\left(K_{B T}\right)^{2}\right)
$$

for finite temperatures.

\subsection{Magnetoconductivity}

The BTE for non-zero magnetic field is realized from (1) by making the transformation $E \rightarrow E+v \times H$ or adding the term $(v \times H) \cdot \nabla g(p)$ in the linearized BTE. This simple replacement will not yield a general solution, because of the cross product. It ensures that $v \cdot(v \times H)=0$. To find the general solution, one usually obtains separate solutions for magnetic and electric fields and superimpose them [12]. Here, we obtained the solution as follows; if the lattice temperature is constant of space, (1) becomes 


$$
\begin{aligned}
& f_{0(p)}+\tau e^{\prime} v \cdot \nabla \Phi\left(-\frac{\partial f(p)}{\partial \varepsilon}\right) \\
& =f(p)+\tau \mathrm{e}(v \times H) \cdot \nabla_{p} f(p) .
\end{aligned}
$$

The electrochemical potential is now defined as $e \nabla \Phi=e^{\prime}\left[\nabla\left(\mu / e^{\prime}\right)-E\right]$, with $e^{\prime}=e /(1-i \Omega \Lambda \mu)$. We have assumed time independent magnetic field. The right hand side of (19) can be seen as an expansion of $f\left(p^{\prime}\right)$, where

$$
p^{\prime}=p+\frac{\tau e v_{F}}{|p|}(p \times H)
$$

so that

$$
f_{0}+\tau e^{\prime} v \cdot \nabla \Phi\left(-\frac{\partial f}{\partial \varepsilon}\right)=f\left(p^{\prime}\right)
$$

We need to invert (20) and put it in (19). To do this, we make $p$ the subject as

$$
p=\frac{1}{1+\eta^{2} H^{2}}\left(p^{\prime}-\eta p^{\prime} \times H\right),
$$

where $\eta=\tau e v_{F} /|p|$. Equation (21) now becomes

$$
f(p)=f_{0}+\frac{\eta}{1+\eta^{2} H^{2}} p \cdot(\nabla \Phi-\eta H \times \nabla \Phi)\left(-\frac{-\partial f}{\partial \varepsilon}\right)
$$

after dropping the prime. Notice the energy dependence of $\eta$ through $\tau$, i.e. $\eta=\alpha \varepsilon^{\beta-1}$. Where $\alpha=\Lambda e v_{F}^{2}$ is identified as the mobility in units of centimeter square per volts per second. Now, to compute the electric current, (23) is used in (4) with $\beta=+1$ to get

$$
J_{e}=\frac{\sigma(\Omega)}{1+\alpha^{2} H^{2}}(\nabla \Phi-\alpha H \times \nabla \Phi) .
$$

The presence of magnetic field vector has created off diagonal elements in the electric current density tensor. To compute the components of the new tensor we write (24) in an indicial notation as

$$
J_{i}^{e}=\frac{\sigma(\Omega)}{1+\alpha^{2} H^{2}}\left(\nabla \Phi_{i}-\alpha \in_{i j k} H_{j} \nabla \Phi_{k}\right) .
$$

The longitudinal and transverse components of the magnetoconductivity tensors are

$$
\begin{gathered}
\sigma_{x x}=\frac{\sigma(\Omega)}{1+\alpha^{2} H^{2}}, \\
\sigma_{x y}=\frac{\sigma(\Omega) \alpha H}{1+\alpha^{2} H^{2}} .
\end{gathered}
$$

The rest of the components are determined through the relations $\sigma_{x x}=\sigma_{y y}$ and $\sigma_{x y}=-\sigma_{y x}$. In terms of magnetoresistivities, (26a) and (26b) are usually written as

$$
\sigma_{x x}=\frac{\rho_{x x}}{\rho_{x x}^{2}+\rho_{x y}^{2}}
$$

$$
\sigma_{x y}=\frac{\rho_{x y}}{\rho_{x x}^{2}+\rho_{x y}^{2}}
$$

with the resistivity $\rho_{x x}=1 / \sigma$ and the Hall resistivity $\rho_{x y}=\alpha H / \sigma$. In terms of electron concentration $n, \rho_{x y}=H / n e$, where $n e=1 / R_{H}$ and $R_{H}$ is the Hall coefficient. In general, $\sigma$ is complex. For this reason, we make the replacement $1 /\left(1+(\alpha H)^{2}\right) \rightarrow 1 /(1-i \alpha H)$ So that both longitudinal and transverse electronic conductivities, in the presence of constant magnetic field, for $\beta=+1$ take the form

$$
\sigma_{x x}(\Omega) / \sigma(0)=\frac{1-(\alpha \mu \Lambda \Omega H)}{\left[1+(\Omega \Lambda \mu)^{2}\right]\left[1+(\alpha H)^{2}\right]}
$$

and

$$
\frac{\sigma_{x y}(\Omega)}{\sigma(0)}=\frac{\mu \Lambda \Omega+\alpha H}{\left[1+(\Omega \Lambda \mu)^{2}\right]\left[1+(\alpha H)^{2}\right]} .
$$

Where the temperature dependent zero frequency conductivity is

$$
\sigma(0)=\frac{\sigma_{\min }}{u_{0}^{2}}\left[\mu^{2}+\frac{\pi^{2}}{3}\left(k_{B} T\right)^{2}\right] .
$$

We now observe the effect of crossed magnetic and electric field on graphene by plotting longitudinal conductivity with frequency and magnetic fields in Figures 3 and 4.

\section{Discussion and Conclusions}

The advantage of our approach is that, one does not need to go through rigorous process of calculating the specific scattering rate $\Gamma(\varepsilon)$. For instance, unlike in [9], finite temperature conductivity was found by separately calculating phonon and normal relaxation times. Quite re-

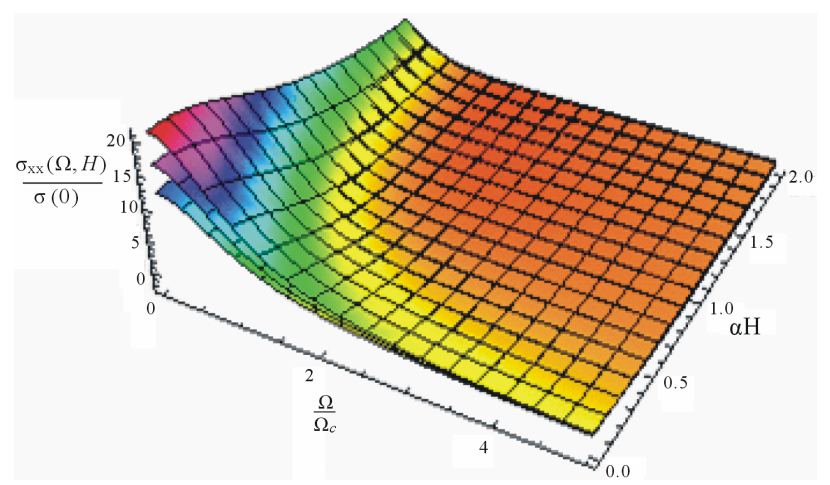

Figure 3. Normalized longitudinal conductivity with $\mathbf{\Omega} / \mathbf{\Omega}_{c}$ and $\alpha H$ at fixed values of doping; Top: $\mu=0.5 \mathrm{eV}$, Middle: $\mu$ $=0.3 \mathrm{eV}$, Bottom: $\mu=0.2 \mathrm{eV}, \mu \geq 2 K_{B} T$ and $\alpha=2.3$ $\mathbf{m}^{2} \cdot \mathbf{V}^{-1} \cdot \mathbf{s}^{-1}$. 


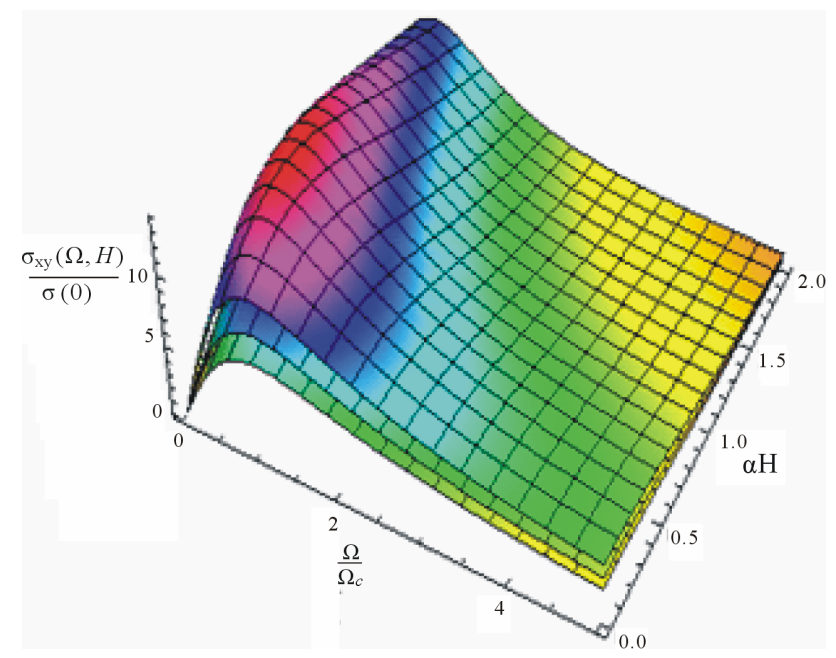

Figure 4. Normalized transverse conductivity is plotted against $\Omega / \Omega_{c}$ and $\alpha H$ at fixed values of doping. Top: $\mu=$ $0.5 \mathrm{eV}$, Middle: $\mu=0.3 \mathrm{eV}$, Bottom: $\mu=0.2 \mathrm{eV}, \mu \geq 2 K_{B} T$ and $\alpha=2.3 \mathrm{~m}^{2} \cdot \mathrm{V}^{-1} \cdot \mathrm{s}^{-1}$.

cently, a specific form of scattering potential was employed for studying scattering processes in graphene superlattice [17]. In this brief article, we obtained similar results without knowing a priori the exact form of the scattering potential. The challenge, however, is that certain material properties (constants, like permittivity), are not integral part of our results. Nonetheless, they can always be found by comparing with literature. But in this report, we do not care so much about numerical values of those constants; we only want to demonstrate the validity and the new physics inherent in our approach. In a static electric field $\Omega=0$, the results obtained agrees well with what was obtained in [9-11,18].

Using phenomenological theory based on semi-classical BTE, we reproduce transport properties of graphene without knowing the type of scattering process. We found that a characteristic exponent of $\beta=+1$ corresponds to charged impurity scattering and is a dominant mechanism in the absence of acoustic phonons. Chemical potential plays an important role in scattering. It directly connects low scattering processes on one hand and dominant scattering processes on the other hand. That is, ballistic is proportional to short-range, $\sigma_{0} \approx \mu \sigma_{-1}$, and acoustic phonon is proportional to long-range coulomb scattering, $\sigma_{+2} \approx \mu \sigma_{+1}$. In Figures 3 and 4, a universal scaling behavior of both conductivities shows up in the regime $\Omega \rightarrow \Omega_{\mathrm{c}}$ and and strong magnetic field, $H \square 1$. That is, $\sigma_{x x} \sim \sigma_{x y} \sim 1 / H$.

The linear energy spectrum employed here may hide some interesting physics, in view of this a further studies could be done using somewhat complex band structure. For instance, a gap spectrum or full tight binding spectrum could be used.

\section{REFERENCES}

[1] A. H. C. Neto, F. Guinea, N. M. R. Peres, K. S. Novoselov and A. K. Geim, "The Electronic Properties of Graphene," Reviews of Modern Physics, Vol. 81, No. 1, 2009, pp. 109-162. doi:10.1103/RevModPhys.81.109

[2] M. I. Katsenelson and K. S. Novoselov, "Graphene: New Bridge between Condensed Matter Physics and Quantum Electrodynamics," Solid State Communications, Vol. 143, No. 1-2, 2007, pp. 3-13. doi:10.1016/j.ssc.2007.02.043

[3] F. Guinea, A. H. C. Neto and N. M. R. Peres, "Electronic States and Landau Levels in Graphene Stacks," Physical Review B, Vol. 73, No. 24, 2006, Article ID: 245426. doi:10.1103/PhysRevB.73.245426

[4] S. Shivaraman, R. A. Barton, X. Yu, J. Alden, L. Herman, M. V. S. Chandrashekhar, J. Park, P. L. McEuen, J. M. Parpia, H. G. Craighead and M. G. Spencer, "FreeStanding Epitaxial Graphene," Nano Letters, Vol. 9, No. 9, 2009, pp. 3100-3105. doi:10.1021/n1900479g

[5] B. Uchoa and A. H. C. Neto, "Superconducting States in Pure and Doped Grapheme," Physical Review Letters, Vol. 98, 2007, Article ID: 146801. doi:10.1103/PhysRevLett.98.146801

[6] S. Adam, E. H. Hwang, V. M Galitski and S. Dar Sarma, "A Self-Consistent Theory for Graphene Transport," Proceedings of the National Academy of Sciences, Vol. 104, No. 47, 2007, pp. 18392-18397. doi:10.1073/pnas.0704772104

[7] S. Adam, E. H. Hwang and S. Das Sarma, "Scattering Mechanisms and Boltzmann Transport in Graphene," Physica E-Low-Dimensional Systems \& Nanostructures, Vol. 40, No. 5, 2008, pp. 1022-1025.

[8] A. K. Geim and K. S. Novoselov, "The Rise of Graphene," Progress Article, Nature Materials, Vol. 6, 2007, pp. 183-191. doi:10.1038/nmat1849

[9] N. M. R. Peres, J. M. B. L. dos Santos and T. Stauber, "Phenomenological Study of the Electronic Transport Coefficients of Graphene," Physical Review B, Vol. 76, No. 7, 2007, Article ID: 073412.

[10] T. Stauber, N. M. R. peres and F. Guinea, "Electronic Transport in Graphene: A Semiclassical Approach Including Midgap States," Physical Review B, Vol. 76, No. 20, 2007, Article ID: 205423.

[11] J. Nilsson, A. H. C. Neto, F. Guinea and N. M. R. Peres, "Electronic Properties of Graphene Multilayers," Physical Review Letters, Vol. 97, No. 26, 2006, Article ID: 266801.

[12] J. M. Ziman and F. R. S. Melville, "Principles of the Theory of Solids," 2nd Edition, Cambridge University Press, Cambridge, 1972.

[13] S. Y. Mensah, F. K. A. Allotey, N. G. Mensah and G. Nkrumah, "Differential Thermopower of a CNT Chiral Carbon Nanotube," Journal of Physics: Condensed Matter, Vol. 13, No. 24, 2001, pp. 5653-5662. doi: $10.1088 / 0953-8984 / 13 / 24 / 310$

[14] O. Madelung, "Introduction to Solid State Theory," Springer-Verlag, Berlin, 1978. doi:10.1007/978-3-642-61885-7

[15] T. Ando and T. Nakanishi, "Impurity Scattering in Car- 
bon Nanotubes-Absence of Back Scattering," Journal of the Physical Society of Japan, Vol. 67, 1998, pp. 17041713. doi:10.1143/JPSJ.67.1704

[16] J.-H. Chen, C. Jang, S. Adam, M. S. Fuhrer, E. D. Williams and M. Ishigami, "Charged-Impurity Scattering in Graphene," Nature Physics, Vol. 4, 2008, pp. 377-381. doi:10.1038/nphys 935
[17] S. V. Kryuchkov and E. I. Kukhar, "Influence of the Magnetic Field onthe Graphene Conductivity," Journal of Modern Physics: Scientific Research, Vol. 3, No. 9, 2012, pp. 994-1001.

[18] K. Namura and A. H. McDonald, "Quantum Hall Ferromagnetism in Graphene," Physical Review Letters, Vol. 96, No. 25, 2006, Article ID: 256602. 